\title{
Hit efficiency study of CMS prototype forward pixel detectors
}

\author{
Dongwook Kim a,1,2 \\ a Johns Hopkins University, Baltimore
}

\begin{abstract}
In this paper I describe the measurement of the hit efficiency of a prototype pixel device for the CMS forward pixel detector. These pixel detectors were FM type sensors with PSI46V1 chip readout. The data were taken with the $120 \mathrm{GeV}$ proton beam at Fermilab during the period of December 2004 to February 2005. The detectors proved to be highly efficient $(99.27 \pm 0.02 \%)$. The inefficiency was primarily located near the corners of the individual pixels.
\end{abstract}

\section{Introduction}

In this paper I describe the analysis of test beam data providing an efficiency measurement for prototypes of the CMS forward pixel detector. Since this is not meant to be a complete write up of the pixel system, I leave out many of the details of the hardware and software which are described elsewhere.

This test was conducted to measure the hit efficiency of the CMS forward pixel detector. A short description of the pixel device is in the appendix. These detectors used the FM type p-stop design shown in Figure 1. This figure shows the layout of the pixels particularly near the corners. A more detailed description of the CMS forward pixel detector can be found in the CMS tracking TDR Ref. (1).

1 Presented at VERTEX2005 on behalf of the CMS Pixel Collaboration.

2 Thanks to Bruce Barnett (Johns Hopkins University) and J. C. Yun (FNAL) for their help on the work described in this paper.

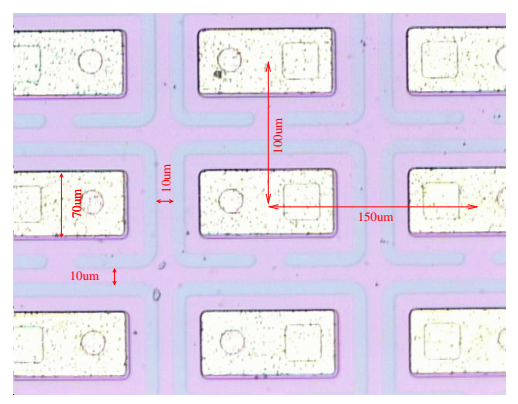

Fig. 1. Diagram of the pixels showing the FM p-stop design

The tracking system used in our FNAL test beam consisted of 8 very high resolution silicon strip planes and some triggering scintillation counters. It was supplied by the Rutgers University CMS group. A very similar system is described in detail in Ref. (1).

This silicon strip tracking system had $4 \mathrm{X}$ and $4 \mathrm{Y}$ views alternating in the beam direction. The appendix gives a brief description of this hardware setup and the DAQ system.

Fermilab provided the $120 \mathrm{GeV}$ proton primary beams which were extracted from the Main Injec- 
tor ring and distributed to the meson beam lines. The test beam setup was located in the MT6 beam line. A short introduction to the test setup is in the appendix.

We took test beam data with pixel bias voltages ranging from $V_{b}=-250 \mathrm{~V}$ to $-400 \mathrm{~V}$ in $50 \mathrm{~V}$ steps. Data was taken with the pixel plane at $90^{\circ}$ to the beam and with the pixel plane rotated at $70^{\circ}$ to the beam. We took about $250 \mathrm{k}$ events at each bias voltage setting and each pixel orientation. For the case of $V_{b}=-350 \mathrm{~V}$, we took more data to increase the statistics of the efficiency measurements. All the data were taken with the pixel device temperature setting of $-20.4^{\circ} \mathrm{C}$.

\section{Efficiency Calculation}

A set of good, well defined data was selected for the efficiency measurements. We applied the following three requirements on the data.

(i) We required all 8 silicon strip planes to have one, and only one, hit cluster in order to select a good quality track. We defined a "strip track" by fitting a line through the 4 strip clusters for both the $\mathrm{X}$ and $\mathrm{Y}$ directions. A good track required that all of the cluster residuals were less than $3 \mu \mathrm{m}$ in this track fit. These cuts reduced the data size to about $30 \%$ of the original triggers.

(ii) We required the track to pass through the fiducial volume of the pixel detector. The cuts for this selection are shown in the Figure 2. The cuts in the $\mathrm{X}$ and $\mathrm{Y}$ directions, which are shown as vertical lines in the figure, were applied to the silicon strip planes. The corresponding lines are shown on the pixel hit distributions.

(iii) We rejected events with questionable electronic readout, specifically bad Token Bit Manager (TBM) trailers. About $1.1 \%$ of the events fell into this category. By studying the data event by event, we learned that sometimes the readout signal turns into a feedback oscillation. The DAQ failed for this case. This situation could be cured by resetting the Read-out Chip (ROC) and the
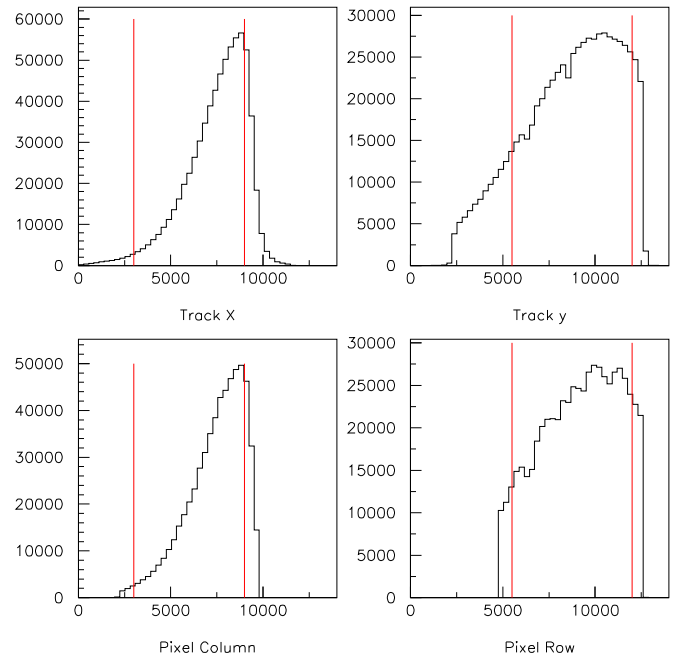

Fig. 2. Hit distributions for the strip planes are shown in upper plots. The bottom plots show hit distributions for the pixel device. The vertical lines show the applied fiducial cuts on the strip hit position. The cuts were not applied to the pixel device.

\begin{tabular}{|c|c|c|}
\hline Items & number of events & efficiency (\%) \\
\hline \hline Total events & $1,000,000$ & \\
\hline Single cluster and seed hit cut & 699,299 & \\
\hline Track residual cut & 483,700 & \\
\hline Pixel fiducial cut & 309,534 & \\
\hline Bad trailer cut & 306,263 & 100.00 \\
\hline Pixel hit cut & 304,990 & $99.58 \pm 0.02$ \\
\hline Track-pixel match cut & 304,022 & $99.27 \pm 0.02$ \\
\hline
\end{tabular}

Table 1

Pixel hit efficiency for successive cuts applied for normal $\left(90^{\circ}\right)$ incident tracks at $V_{b}=-350 \mathrm{~V}$. Statistical errors only.

TBM. Also, the DAQ settings allowed at most 7 pixel hits in an event. Sometimes, there were more than 7 triggered pixels in the event, in which case the event data was chopped off and, hence, was missing the trailer. Occasionally, the trailer analog levels were too far off from the nominal value and missed the predetermined cut. These events were not used in the efficiency study. 


\begin{tabular}{|c|c|c|c|}
\hline Bias Voltage & Total events & Good track & Good pixel (Efficiency \%) \\
\hline \hline-200 & 250,000 & 70,348 & $68,002(96.67 \pm 0.07 \%)$ \\
\hline-250 & 250,000 & 76,221 & $75,553(99.12 \pm 0.04 \%)$ \\
\hline-300 & 250,000 & 70,868 & $70,394(99.33 \pm 0.03 \%)$ \\
\hline-350 & $1,000,000$ & 306,263 & $304,022(99.27 \pm 0.02 \%)$ \\
\hline-400 & 250,000 & 70,370 & $69,185(98.32 \pm 0.05 \%)$ \\
\hline
\end{tabular}

Table 2

Pixel hit efficiency versus bias voltage for the normal incident $\left(90^{\circ}\right)$ tracks. Statistical error only.

Using this selected data sample we measured the pixel hit efficiency by requiring pixel hits aligned with the silicon system tracks. About $0.4 \%$ of all the events did not have any signals from the pixels. For a pixel signal to be counted as a "hit" the pixel's center had to be within a window of $\pm 85 \mu \mathrm{m}$ in $\mathrm{X}$ and $\pm 60 \mu \mathrm{m}$ in $\mathrm{Y}$ centered around the interpolated strip track position at the pixel plane. The additional inefficiency, i.e. the probability of there being no hit pixel within this window for good silicon tracks, was about $0.3 \%$. The probability of a random pixel hit matching the silicon track was negligible.

Table 1 shows the cuts and resulting data reduction for the $90^{\circ}$ data taken with the pixel bias voltage at $V_{b}=-350 \mathrm{~V}$. The hit efficiency is measured to be $99.27 \pm 0.02 \%$, where the errors are statistical only.

In Figure 3 I show the efficiency distributions for various bias voltage settings. The horizontal scale is in pixel units, i.e. " 0 " is the center of the gap between two adjacent pixels while +0.5 and -0.5 are at the centers of the two pixels. A large dip in the efficiency is visible at the gap between the pixels when the bias voltage is $V_{b}=-200 \mathrm{~V}$. The dip is smaller and nearly constant for bias voltages lower than $V_{b}=-250 \mathrm{~V}$. Judging from these plots, the bias voltage is fully sufficient when it is lower than $V_{b}=-250 \mathrm{~V}$.

Figure 4 shows the hit inefficiencies for the $90^{\circ}$ data. In this scatter plot, each dot represents the position of strip track interpolated to the pixel plane when there is no matching pixel hit. The dense area in the middle of the distribution is where the corners of 4 pixels meet. From this plot we conclude that most of the inefficiency is coming from
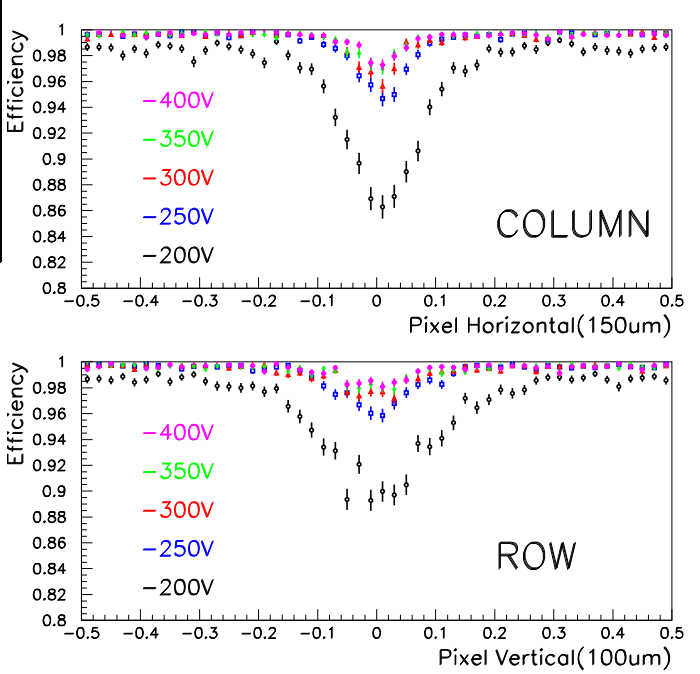

Fig. 3. Efficiency plots for the pixel device for the column (top) and row (bottom) directions for the normal incident $\left(90^{\circ}\right)$ tracks. The horizontal scale is in pixel units, i.e. the horizontal axis is the distance from the middle point of two adjacent pixels. The error bars on the plots are statistical only.
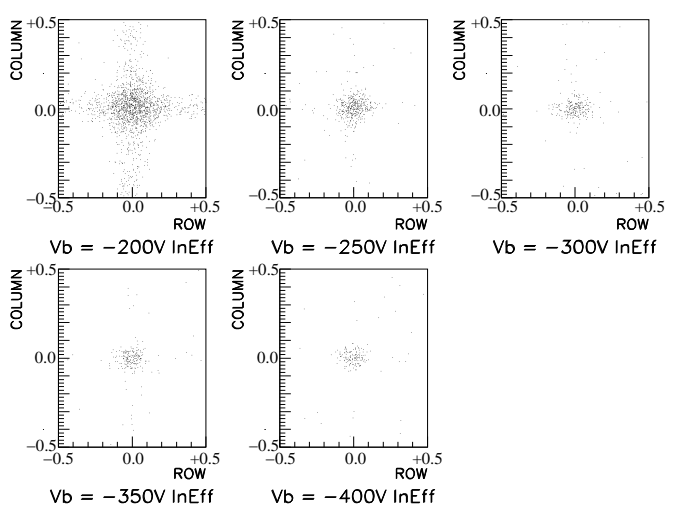

$\mathrm{Vb}=-300 \mathrm{~V} \ln \mathrm{E} f \mathrm{f}$

Fig. 4. Two dimensional inefficiency distributions for various bias voltages for normal incident $\left(90^{\circ}\right)$ tracks. Each dot represents the position of a strip track interpolated to the pixel plane when there is no matching pixel hit. The center of the plot is where the corners of 4 pixels meet. When the bias voltage is $V_{b}=-200 \mathrm{~V}$ the vertical and horizontal gaps between the pixels are clearly seen as an inefficiency in the distribution. 


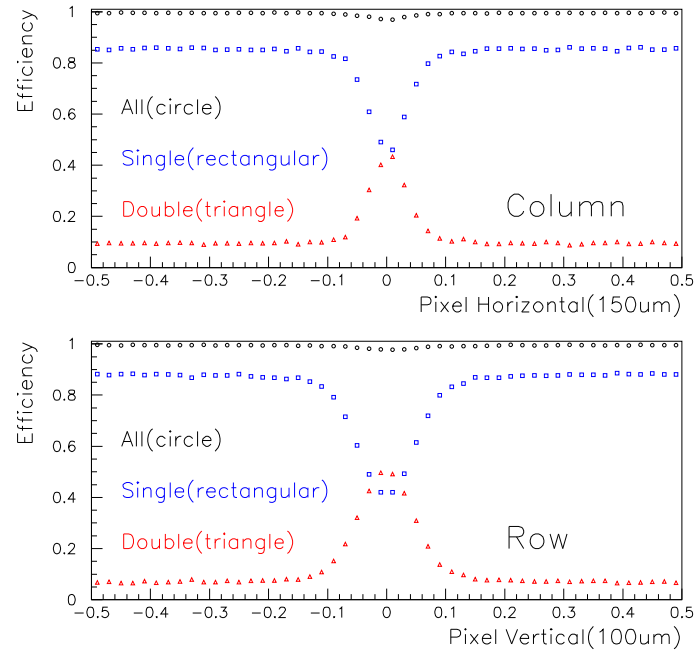

Fig. 5. Relative efficiency plots between single hit and double hits case for the pixel device for the column (top) and row (bottom) directions for the normal incident $\left(90^{\circ}\right)$ tracks. The horizontal scale is in pixel units, i.e. the horizontal axis is the distance from the middle point of two adjacent pixels. The error bars on the plots are statistical only.

this spot.

We studied the efficiency of the detectors separately for cases where there was a single hit pixel and for cases where there were two hit pixels. The results of these studies are shown in Figure 5 for the two cases of scanning over the pixels' columns and rows. One sees that the single hit efficiencies are high when one is far from the pixels' edge and dip if the particle passes near the edge, whereas the double hit efficiency is small when the particle is far from the edge and peaks when the particle is near the edge. This shows the effects of charge sharing for neighboring pixels. The sum of the two efficiencies, what we call the real efficiency of the pixels, is also shown in the figure and is relatively flat with a small dip when the particle passes through the region between the pixels.

We also studied the effect of the track's incident angle to the pixel plane. In the CMS experiment the pixels will be rotated such that the incident angle for the tracks coming from the interaction point is $70^{\circ}$. This is intended to enhance the po-

\begin{tabular}{|c|c|c|}
\hline Items & number of events & efficiency (\%) \\
\hline \hline Total events & 500,000 & \\
\hline Single cluster and seed hit cut & 341,514 & \\
\hline Track residual cut & 242,884 & \\
\hline Pixel fiducial cut & 161,405 & \\
\hline Bad trailer cut & 159,492 & 100.00 \\
\hline Pixel hit cut & 158,905 & $99.63 \pm 0.02$ \\
\hline Track-pixel match cut & 158,407 & $99.32 \pm 0.02$ \\
\hline
\end{tabular}

Table 3

Pixel hit efficiencies as successive cuts are applied for the $70^{\circ}$ incident track data at $V_{b}=-350 \mathrm{~V}$. Statistical errors only.

\begin{tabular}{|c|c|c|c|}
\hline Bias Voltage (V) & Total events & Good track & Good pixel (Efficiency\%) \\
\hline \hline-200 & 250,000 & 74,938 & $63,005(84.04 \pm 0.14 \%)$ \\
\hline-250 & 250,000 & 75,618 & $75,013(99.20 \pm 0.04 \%)$ \\
\hline-300 & 250,000 & 71,511 & $71,046(99.35 \pm 0.03 \%)$ \\
\hline-350 & 500,000 & 159,492 & $158,407(99.32 \pm 0.02 \%)$ \\
\hline-400 & 250,000 & 73,734 & $73,310(99.43 \pm 0.03 \%)$ \\
\hline
\end{tabular}

Table 4
Pixel hit efficiency table for various bias voltages for the $70^{\circ}$ incident tracks. Statistical errors only.

sition resolution by increasing the charge sharing between neighboring pixels. To measure this effect we rotated the pixel plane in the test beam by 20 degrees to make the incident angle $70^{\circ}$ to the column direction. This rotation should increase the charge sharing for neighboring columns but have little effect on neighboring rows.

The Table 3 shows the effect of various cuts on the data for the $70^{\circ}$ incident angle and bias voltage $V_{b}=-350 \mathrm{~V}$. The efficiency values changed very little from the case of $90^{\circ}$ incidence. The Table 4 shows the efficiency results for the bias voltage scan of the $70^{\circ}$ data.

The analysis of the $70^{\circ}$ data is summarized in three figures. Figure 6 shows the efficiency plots for the $70^{\circ}$ incident angle. The $70^{\circ}$ data shows a smaller and wider dip in the column data than the $90^{\circ}$ data. Figure 7 shows the scatter plot for the $70^{\circ}$ incident angle. The densely populated area is elongated along the direction of column when compared to the Figure 4 . When charge sharing occurs the probability of having charge below threshold 

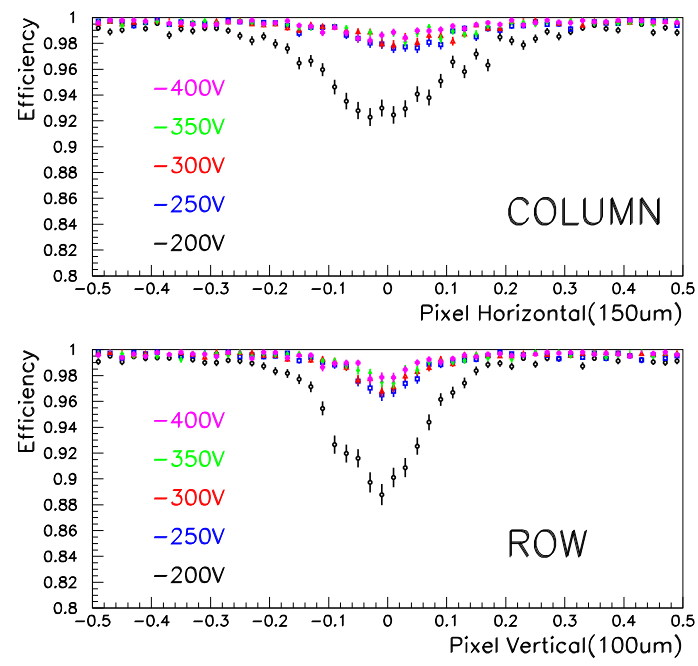

Fig. 6. Efficiency plots for the pixel device for column (top) and row (bottom) directions for the $70^{\circ}$ incident tracks. The horizontal scale is in pixel units, i.e. the horizontal axis is the distance from the mid point of two adjacent pixels. The error bars on the plots are statistical only.
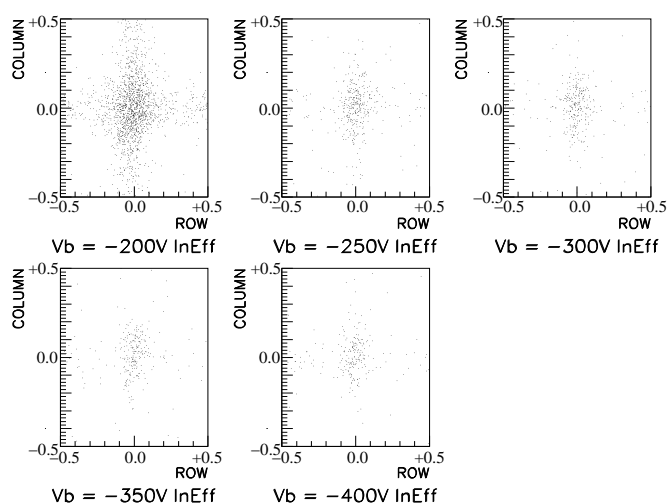

$\mathrm{Vb}=-350 \mathrm{~V} \ln \mathrm{Eff}$

$\mathrm{Vb}=-400 \mathrm{~V} \operatorname{In} E f f$

Fig. 7. Two dimensional inefficiency distributions for various bias voltages for $70^{\circ}$ incident tracks. Each dot records the position of a strip track extrapolated to the pixel plane when there is no matching pixel hit. The center of the plot is where the corners of 4 pixels meet. When the bias voltage is $V_{b}=-200 \mathrm{~V}$, the vertical and horizontal gaps between the pixels are clearly seen in the distribution. This is where the edges of two neighboring pixels meet.
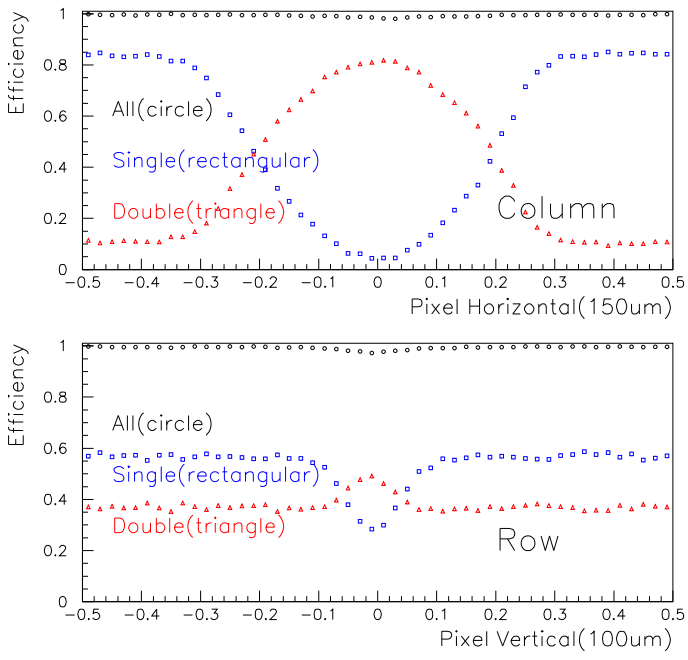

Fig. 8. Efficiency plots between single hit and double hits case for the pixel device for column (top) and row (bottom) directions for the $70^{\circ}$ incident tracks. The horizontal scale is in pixel units, i.e. the horizontal axis is the distance from the mid point of two adjacent pixels. The error bars on the plots are statistical only.

becomes relatively larger and the pixels' hits trigger less frequently. Figure 8 shows the single and double hit efficiencies of the $70^{\circ}$ data for the two cases of scanning over the columns and over the rows. One sees a behavior similar to that for the $90^{\circ}$ data in Figure 5 except that for the row data the single hit efficiency is lower and the double hit efficiency is higher in the regions far from the pixel edges and the single hit dip and double hit peak is smaller near the edge. This difference between the scan along the column and scan along the row clearly shows the effect of the tilting.

\section{Conclusion}

We measured the efficiency of the CMS prototype forward pixel device of the Sintef FM design with a PSI46V1 chip readout. The efficiency is $99.27 \pm 0.02 \%$ for a bias voltage of $V_{b}=-350 \mathrm{~V}$. We observe that most of the inefficiency occurs where the corners of 4 pixels meet. Very little change in total efficiency was observed for the case of $70^{\circ}$ 
incident angle (99.32 $\pm 0.02 \%)$ although the inefficiency was a bit more spread out around the gap. The extra charge sharing in the $70^{\circ}$ data should improve the spatial resolution of the pixels, but we did not measure that.

\section{References}

[1] CMS Collaboration, Tracker Technical Design Report, CERN/LHCC 98-6, April 15, 1998.

[2] Design and Test of Pixel Sensor for CMS Experiment, A, Roy, NIM A461 (2001) 416

[3] Readout Architectures for pixel detectors, R. Horisberger, NIM A465 (2000) 148

[4] A submicron precision silicon telescope for beam test purposes, C. Colledani et al., NIM A372 (1996) 379-384

\section{Appendix A. Pixel Device}

A pixel device consists of a sensor, a ROC (read out chip, PSI46V1), a VHDI (Very High Density Interface) and HDI (High Density Interface). The signals are read out through a TBM (token bit manager). The single pixel size is $100 \mu \mathrm{m}$ center to center in the row direction and $150 \mu \mathrm{m}$ center to center in the column direction. A ROC has 80 rows and 52 columns. Each pixel is bump bonded to a matching pad on the ROC. Details of pixel device design and readout can be found in the Ref. (2), (3).

The PSI46V1 ROC does not provide charge information. For an efficiency study this is sufficient since a pixel triggers the readout when the charge collected in the pixel is above a set charge threshold.

During data taking the pixel device resided in a box, called a "cartridge", which was made from foam material to provide insulation. Dry nitrogen gas was constantly flowing through the cartridge to prevent water condensation on the pixel surface.
A Peltire cooling system was attached to the pixel chip to cool it down to the operating temperature of $-20.4^{\circ} \mathrm{C}$.

\section{Appendix B. Silicon Strip Planes}

There are 256 channels in each silicon strip plane. The strip center to center distance was $50 \mu \mathrm{m}$. After a simple alignment with rotational and translational correction from beam data, a typical sigma for the deviation distribution was $2.5 \mu \mathrm{m}$. This corresponded to a track resolution of about $3.5 \mu \mathrm{m}$ at the pixel plane. A deviation distribution plot is shown in Figure B.1 where a simple linear least square fit was made using the cluster position.

The pixel detector and the 8 strip planes were mounted on a movable table which could be remotely controlled in the $\mathrm{X}$ and $\mathrm{Y}$ directions. Triggering scintillation counters were also in the beamline for strict timing purposes. A schematic diagram of the system is shown in Figure B.2. A group of 4 strip planes were upstream of the pixels and the other group of 4 were located downstream of the pixels. The strip planes were arranged in such a way that the $\mathrm{Y}$-view and $\mathrm{X}$-view planes were alternating in the $\mathrm{Z}$ position. The distance between first and the last silicon strip plane was $61.5 \mathrm{~cm}$.

The pixel detector was mounted at the center of a plate which could be easily rotated and aligned to a desired incident angle by inserting a dowel pin into precision index holes.

\section{Appendix C. MT6 Beamline}

The MT6 beamline provided $120 \mathrm{GeV}$ protons extracted from the Main Injector ring. The beams were delivered in 6 to 12 spills every minute. The typical coincidence rate for the scintillation counters was a few thousand per spill. We read out only those events with hits in the silicon strip planes, which reduced the DAQ readout rate reduces to about 300 events per spill. 

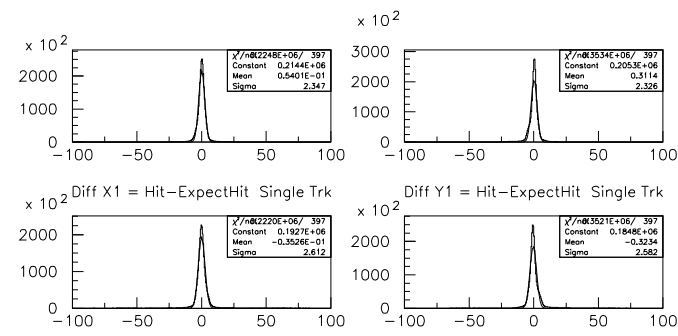

Diff $Y 1=$ Hit-ExpectHit Single Trk
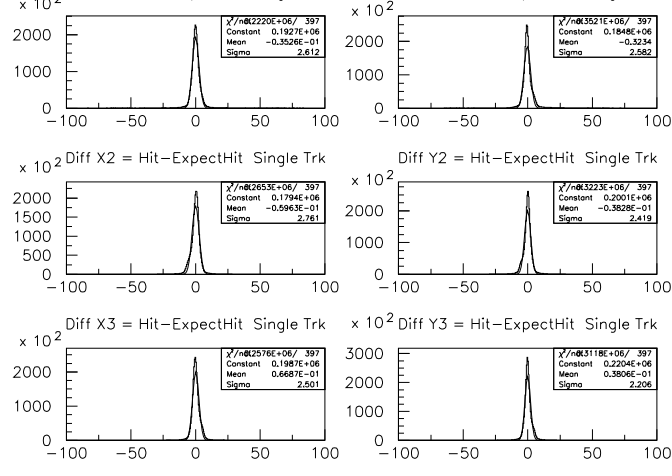

Diff $X_{4}=$ Hit-ExpectHit Single Trk

Diff $Y 4=$ Hit - ExpectHit Single Trk

Fig. B.1. Track residuals of each strip plane from a simple linear fit to the strip clusters for the selected tracks.
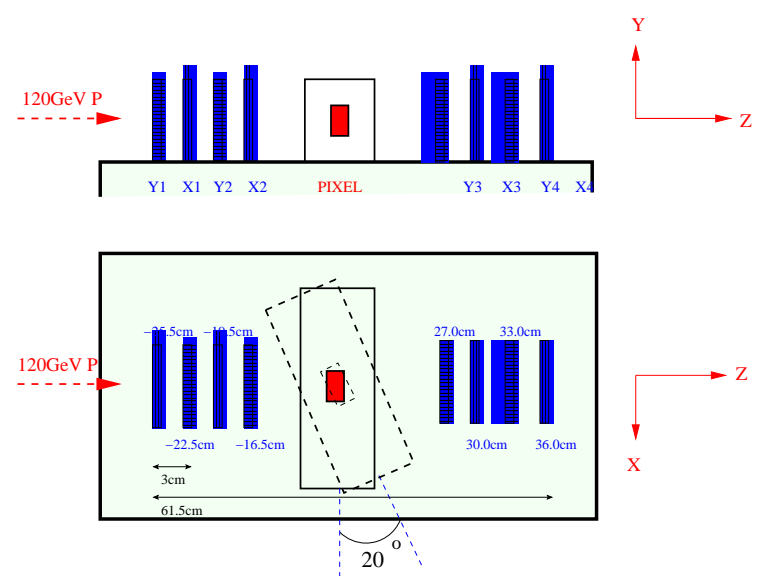

Fig. B.2. There were 4 upstream silicon strip planes and 4 downstream silicon strip planes. The pixel device was located in the middle of the gap. The distance between two neighboring planes was $3 \mathrm{~cm}$. The middle gap is $43.5 \mathrm{~cm}$ wide. 